\title{
A one-pot synthesis of highly substituted tetrahydropyridines from $N$-benzylidenemethanamines, dialkyl acetylenedicarboxylates and benzylidenemalononitriles
}

\author{
Hossein Mehrabi* and Ahmad Mohebbi \\ Department of Chemistry, Vali-e-Asr University of Rafsanjan, 77176 Rafsanjan, Iran \\ E-mail:mehraby_h@yahoo.com
}

DOI: $\underline{\text { http://dx.doi.org/10.3998/ark.5550190.p009.701 }}$

\begin{abstract}
A simple and efficient synthesis of highly substituted tetrahydropyridines has been developed using a one-pot, two step reactions. The synthesis of a series of $N$-benzylidenemethanamines from benzylamines and benzaldehydes in the presence of acetic acid, followed by cyclization with dialkyl acetylenedicarboxylates and benzylidenemalononitriles, gave the corresponding tetrahydropyridines in good yields.
\end{abstract}

Keywords: Tetrahydropyridines, $N$-benzylidenemethanamines, dialkyl acetylenedicarboxylates, benzylidenemalononitriles

\section{Introduction}

Tetrahydropyridines are one of the fundamental heterocycles, which have been the subject of intense research for their outstanding biological properties and wide range of applications to pharmaceutical compounds and synthetic intermediates. ${ }^{1-8}$ Tetrahydropyridine derivatives are also useful against several metabolic disorders and human ailments. The prominent biological activities associated with this pharmacophore are antiparasitic, antimicrobial, anticancer and antiviral. Further, these are intricately involved in MAO based mechanism in Parkinson's disease, ${ }^{2-13}$ as inhibitors of farnesyl transferase, ${ }^{14}$ dihydroorate dehydrogenase ${ }^{15,16}$ and also play key roles in many disease processes.

The first synthesis of dihydropyridines via a cyclocondensation reaction of acetoacetic ester, aldehyde and ammonia was reported by Arthur Hantzsch in $1882 .{ }^{17}$ Multi-component reactions (MCRs) have emerged as a powerful synthetic tool in organic synthesis due to their advantages over the conventional multi-step synthesis. ${ }^{18}$ Therefore, an strategy for the synthesis of the functionalized tetrahydropyridines is using MCRs. One of the most generally used approaches for the synthesis of this class of compounds is three-component reactions of anilines, aromatic aldehydes and acetoacetates in the presence of L-proline/TFA, ${ }^{19}$ bromodimethylsulfonium 
bromide (BDMS), ${ }^{20}$ tetrabutylammonium tribromide (TBATB), ${ }^{21}$ molecular iodine, ${ }^{22}$ indium chloride $\left(\mathrm{InCl}_{3}\right){ }^{23}$ zirconium oxy chloride $\left(\mathrm{ZrOCl}_{2}\right),{ }^{24}$ ceric ammonium nitrate $(\mathrm{CAN})^{25}$ and silica-supported boron trifluoride $\left(\mathrm{BF}_{3} \cdot \mathrm{SiO}_{2}\right) \cdot{ }^{26}$ Four-component reactions of acetylenedicarboxylates, malononitrile, aldehydes and amines in the presence of polyethylene glycol (PEG), ${ }^{27}$ L-proline/ $\mathrm{FeCl}_{3}{ }^{28}$ and the base conditions, ${ }^{29}$ is also reported to give tetrahydropyridines. The reaction of Meldrum's acid, acetoacetates, aromatic aldehydes and ammonium acetate by using Lawesson's reagent under microwave irradiation is also known to give tetrahydropyridines. ${ }^{30}$ Although these methods have their own merits, but suffer from the drawbacks with respect to cost of reagents, reaction conditions, and reaction work-ups. Consequently, this tandem reaction still requires an efficient protocol for the synthesis of tetrahydropyridine derivatives. Herein we report a one-pot, two-step synthesis of substituted tetrahydropyridines via benzylamines and aldehydes with dialkyl acetylenedicarboxylates and benzylidenemalononitriles as starting materials under catalyst-free conditions.

\section{Results and Discussion}

We began our investigation with the construction of $N$-benzylidenemethanamines, which are versatile synthetic intermediates, from benzylamines and benzaldehyde derivatieves (Table 1). The initial reaction was attempted using benzylamine (1a) and benzaldehyde (2a) (Table 1, entry 1). When 1a and 2a were treated in the presence of two drops acetic acid in acetonitrile at room temperature, $N$-benzylidenemethanamine (3a) was obtained in $85 \%$ yield. Various $N$ benzylidenemethanamines 3 were prepared in good yields as shown in Table 1.

After the successful preparation of $N$-benzylidenemethanamines 3a-3f, and with the aim to construct substituted tetrahydropyridines, we selected $\mathbf{3 a}$ as a model compound to examine its reactions with a dimethyl acetylenedicarboxylate and a benzylidenemalononitrile under different conditions. Upon treatment of 3a with dimethyl acetylenedicarboxylate (4a) and 2benzylidenemalononitrile (5a) in acetonitrile solvent, the reaction furnished the desired product, dimethyl 1-benzyl-5,5-dicyano-1,4,5,6-tetrahydro-4,6-diphenylpyridine-2,3-dicarboxylate (6a) (Scheme 1). 
Table 1. Synthesis of $N$-benzylidenemethanamines<smiles>[R]c1ccccc1/C=N/Cc1ccc([R])c(C(=O)OC(C)(C)C)c1</smiles>

\begin{tabular}{|c|c|c|c|c|}
\hline Entry & $\mathrm{R}_{1}$ & $\mathrm{R}_{2}$ & Product & Yield $(\%)^{\mathrm{a}}$ \\
\hline 1 & $\mathrm{H}$ & $\mathrm{H}$ & & 85 \\
\hline 2 & $\mathrm{H}$ & 4-OMe & & 82 \\
\hline 3 & $\mathrm{OMe}$ & $\mathrm{H}$ & & 88 \\
\hline 4 & $\mathrm{Cl}$ & $\mathrm{H}$ & & 80 \\
\hline 5 & $\mathrm{Cl}$ & $2-\mathrm{Cl}$ & & 78 \\
\hline 6 & $\mathrm{H}$ & $2-\mathrm{Cl}$ & & 80 \\
\hline
\end{tabular}

${ }^{a}$ Yields are given for isolated product

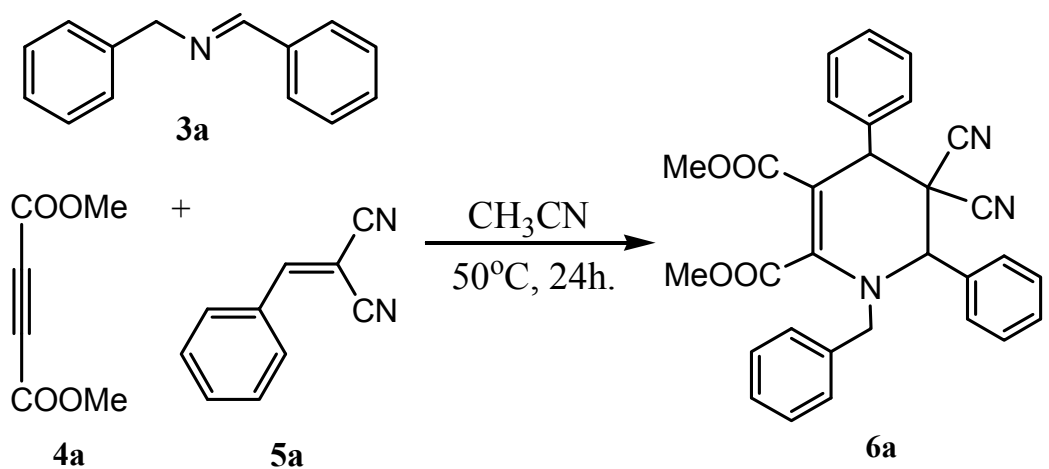

Scheme 1. The optimization of the model reaction. 
The optimization of the reaction conditions, including mmol of starting mterials, reaction solvent, temperature, and the time of reaction, was then investigated. First, solvent-free conditions and various solvent were screened (Table 2, entries 1-6), with acetonitrile proving to be the best for this reaction. Then, we examined the influence of different temperature on this reaction. To our satisfaction, when the reaction was carried out at room temperature in $24 \mathrm{~h}$, the product was $62 \%$ yield but at $50{ }^{\circ} \mathrm{C}$ and reflux conditions in the same time the product was $75 \%$ and $70 \%$ yield respectively (Table 2, entries 7-9). Finally, we observed that the ratio of starting materials also had an important influence on the reaction, a more amount of $\mathbf{4 a}$ (for example, 1.5 $\mathrm{mmol}$ ) in acetonitrile at $50^{\circ} \mathrm{C}$ resulted in a more yield, $80 \%$ (Table 2, entries 9-11).

Table 2. Optimization of the synthesis of highly substituted tetrahydropyridines

\begin{tabular}{ccccc}
\hline Entry & 3a:4a:5a & Solvent/Temp $\left({ }^{\circ} \mathrm{C}\right)$ & Time $(\mathrm{h})$ & Yield $^{\mathrm{a}}(\%)$ \\
\hline 1 & $1: 1: 1$ & Solvent-free, rt & 12 & 0 \\
2 & $1: 1: 1$ & $\mathrm{MeOH}, \mathrm{rt}$ & 12 & Trace \\
3 & $1: 1: 1$ & EtOH, rt & 12 & Trace \\
4 & $1: 1: 1$ & $\mathrm{THF}, \mathrm{rt}$ & 12 & Trace \\
5 & $1: 1: 1$ & $\mathrm{DCM}, \mathrm{rt}$ & 12 & 30 \\
6 & $1: 1: 1$ & $\mathrm{MeCN}, \mathrm{rt}$ & 12 & 50 \\
7 & $1: 1: 1$ & $\mathrm{MeCN}, \mathrm{rt}$ & 24 & 62 \\
8 & $1: 1: 1$ & $\mathrm{MeCN}, 50^{\circ} \mathrm{C}$ & 24 & 75 \\
9 & $1: 1: 1$ & $\mathrm{MeCN}, \mathrm{reflux}$ & 24 & 70 \\
10 & $1: 1.2: 1$ & $\mathrm{MeCN}, 50^{\circ} \mathrm{C}$ & 24 & 77 \\
11 & $1: 1.5: 1$ & $\mathrm{MeCN}, 50^{\circ} \mathrm{C}$ & 24 & 80 \\
\hline
\end{tabular}

${ }^{a}$ Yields are given for isolated product.

With the optimized conditions in hand (Table 2, entry 11), we next investigated the possibility of the synthesis of substituted tetrahydropyridines via a one-pot reaction from benzylamines and aldehydes with dialkyl acetylenedicarboxylates and benzylidenemalononitriles. To explore the scope of this novel transformation, various benzylamines, aldehydes, dialkyl acetylenedicarboxylates and benzylidenemalononitriles were utilized under the optimized one-pot conditions. The results in Table 3 show that all the reactions proceeded smoothly to afford the diastereoisomeric substituted tetrahydropyridines (cis-6 and trans-6) in good yields. Of course, we could not to separate the diastereoisomers from the mixture of reaction by column chromatography.

The NMR spectra of the resulting mixture clearly showed the presence of two the diastereoisomeric cis-6 and trans-6. The ratio of isomers was determined from the intensities of specific signals in ${ }^{1} \mathrm{H}$ NMR spectra. For instance, the ${ }^{1} \mathrm{H}$ NMR spectrum of the compound $6 \mathbf{a}$ consisted of two singlet signals at $\delta 3.32$ (3H, Minor isomer) and $\delta 3.51$ (5H, Major isomer) ppm due to the methoxy groups, one singlet signal at $\delta 3.88(3 \mathrm{H}$, Minor isomer) ppm for the methoxy 
group, a multiplet at $\delta 3.97-4.08$ (9H, Major/Minor isomers) ppm due to the methoxy group, the $\mathrm{CH}$ chiral proton and the $\mathrm{CH}_{2}-\mathrm{Ph}$ methylene protons, a multiplet at $\delta 4.36-4.54(6 \mathrm{H}$, Major/Minor isomers) ppm for the $\mathrm{CH}$ chiral proton and the $\mathrm{CH}_{2}$ - $\mathrm{Ph}$ methylene protons, and a multiplet at $\delta 6.98-7.42(39 \mathrm{H}$, Major/Minor isomers) ppm for the aromatic protons of the phenyl rings. The ${ }^{1} \mathrm{H}$ decoupled ${ }^{13} \mathrm{C}$ NMR spectrum of compound 6a showed 41 distinct signals in agreement with the proposed two isomer structure that characteristic 12 signals for the chiral, benzylic, and methoxy carbons at $\delta 29.71-63.29 \mathrm{ppm}, 5$ signals for cyanide and alkene carbons at $\delta 96.19-113.84 \mathrm{ppm}, 20$ signals due to the aromatic carbons of the phenyl rings at $\delta 128.23$ $149.91 \mathrm{ppm}$, and 4 signals for the carbonyl carbons at $\delta 165.01-165.81 \mathrm{ppm}$.

Table 3. Synthesis of highly substituted tetrahydropyridines

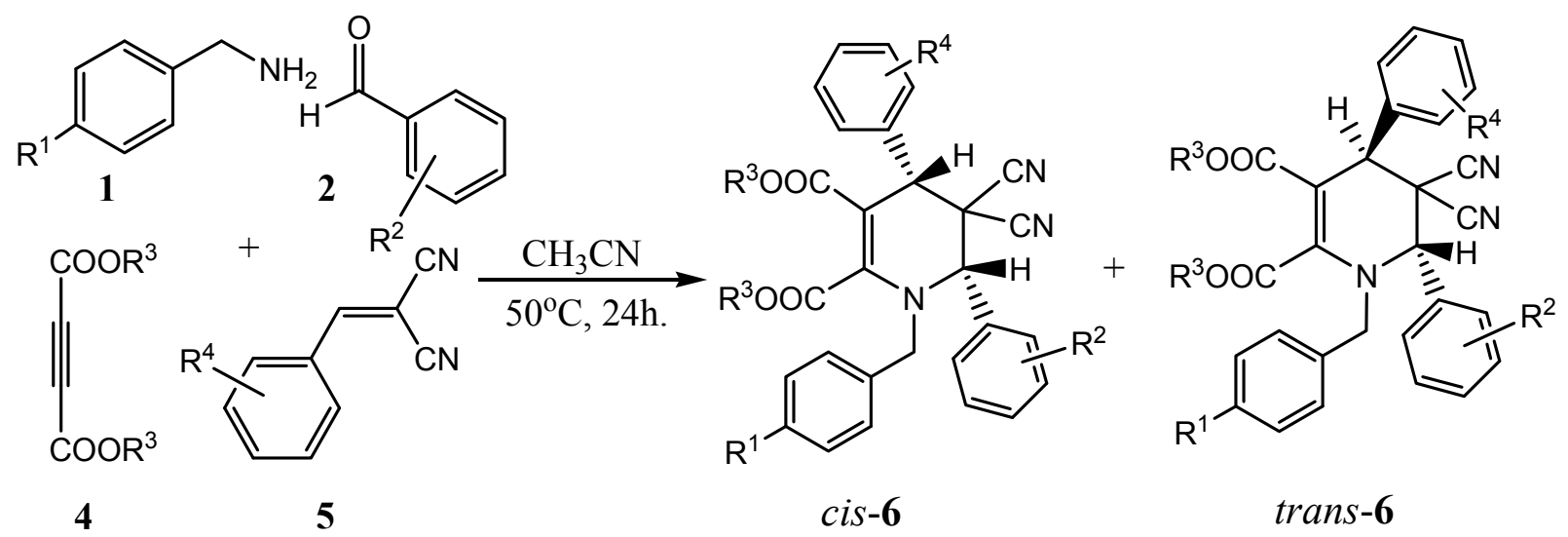

\begin{tabular}{cccccccc}
\hline Entry & $\mathrm{R}^{1}$ & $\mathrm{R}^{2}$ & $\mathrm{R}^{3}$ & $\mathrm{R}^{4}$ & Product & Yield $^{\mathrm{a}}(\%)$ & Major/Minor \\
\hline 1 & $\mathrm{H}$ & $\mathrm{H}$ & $\mathrm{Me}$ & $\mathrm{H}$ & $\mathbf{6 a}$ & 80 & $1.7: 1.0$ \\
2 & $\mathrm{H}$ & $\mathrm{H}$ & $\mathrm{Me}$ & $4-\mathrm{OMe}$ & $\mathbf{6 b}$ & 73 & $2.0: 1.0$ \\
3 & $\mathrm{H}$ & $\mathrm{H}$ & $\mathrm{Me}$ & $4-\mathrm{NO}_{2}$ & $\mathbf{6 c}$ & 77 & $3.3: 1.0$ \\
4 & $\mathrm{H}$ & $4-\mathrm{OMe}$ & $\mathrm{Me}$ & $\mathrm{H}$ & $\mathbf{6 d}$ & 72 & $1.8: 1.0$ \\
5 & $\mathrm{OMe}$ & $\mathrm{H}$ & $\mathrm{Me}$ & $2-\mathrm{Cl}$ & $\mathbf{6 e}$ & 83 & $1.7: 1.0$ \\
6 & $\mathrm{H}$ & $\mathrm{H}$ & $\mathrm{Me}$ & $4-\mathrm{Cl}$ & $\mathbf{6 f}$ & 85 & $1.9: 1.0$ \\
7 & $\mathrm{Cl}$ & $\mathrm{H}$ & $\mathrm{Me}$ & $\mathrm{H}$ & $\mathbf{6 g}$ & 75 & $1.6: 1.0$ \\
8 & $\mathrm{Cl}$ & $2-\mathrm{Cl}$ & $\mathrm{Me}$ & $4-\mathrm{NO}_{2}$ & $\mathbf{6 h}$ & 82 & $2.5: 1.0$ \\
9 & $\mathrm{H}$ & $2-\mathrm{Cl}$ & $\mathrm{Et}$ & $\mathrm{H}$ & $\mathbf{6 i}$ & 85 & $2.2: 1.0$ \\
10 & $\mathrm{Cl}$ & $\mathrm{H}$ & $\mathrm{Et}$ & $2-\mathrm{Cl}$ & $\mathbf{6 j}$ & 75 & $2.4: 1.0$ \\
\hline
\end{tabular}

${ }^{\mathrm{a}}$ Yields are given for isolated product.

A mechanistic rationalization for the reaction is given in Scheme 2. nucleophilic attack of $\mathbf{3}$ on the electron-deficient triple bond $\mathbf{4}$ could lead to the formation of intermediate $\mathbf{A}$, which possessed a resonance structure $\mathbf{B}$ as a 1,4-dipolar synthon. When the benzylidenemalononitrile $\mathbf{5}$ existed in the mixture of $\mathbf{3}$ and $\mathbf{4}$, a subsequent [4+2] cycloaddition occurred. In this way, the 
corresponding substituted tetrahydropyridine derivatives 6 were prepared. During the process of the cycloaddition, there are four possible transition states $\mathbf{C}, \mathbf{D}, \mathbf{E}$, and $\mathbf{F}$, which might lead to the formation of four possible products, cis-6 and trans-6 accordingly. C presented the lowest energy while $\mathbf{F}$ had the highest energy.<smiles>[R20]C(F)(F)CN(C[Ga])CCC(C)C</smiles>

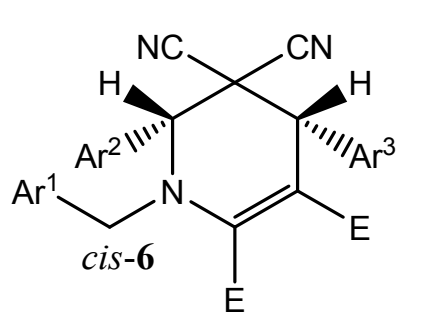

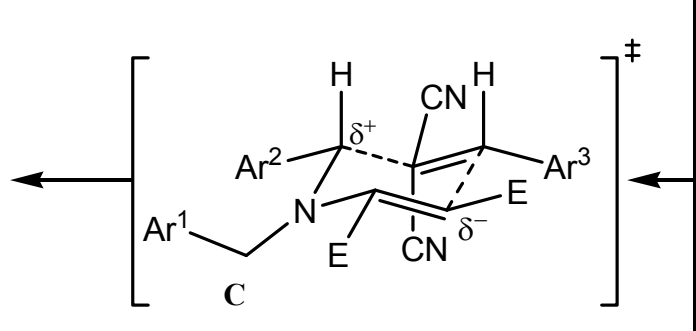

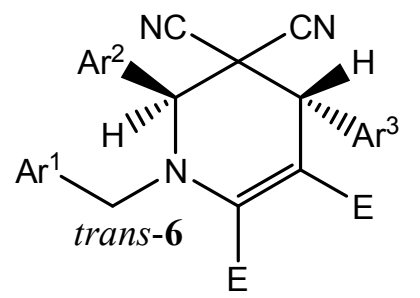

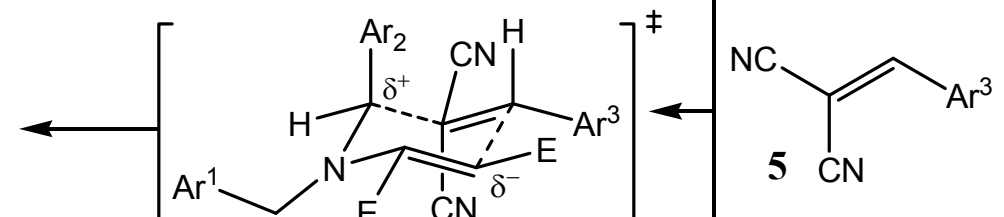

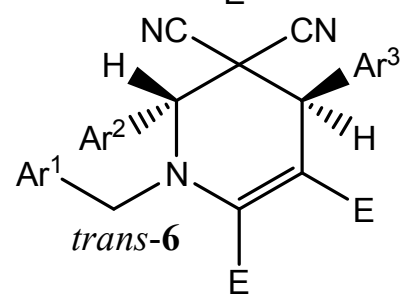

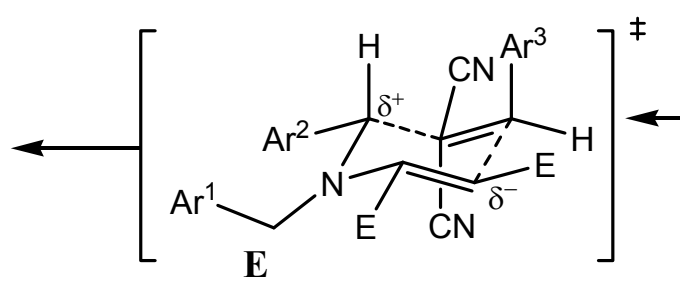

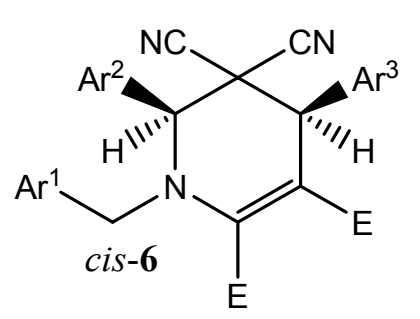

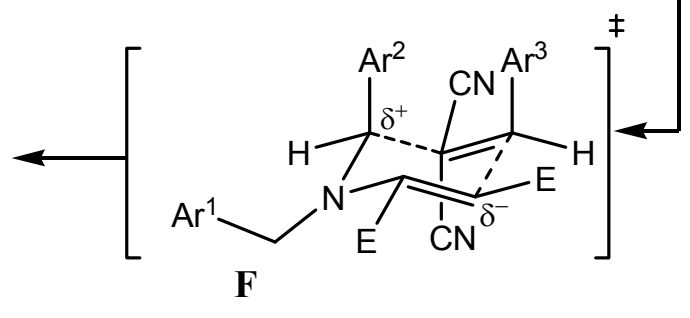

Scheme 2. Proposed mechanism for the synthesis of highly substituted tetrahydropyridines. 


\section{Conclusions}

We have developed an efficient and facile method for the synthesis of highly substituted tetrahydropyridine derivatives by treatment of $N$-benzylidenemethanamines, dialkyl acetylenedicarboxylates, and benzylidenemalononitriles under catalyst-free conditions. The mild reaction conditions, low cost of the starting materials, operational simplicity and good yields are advantages of the protocol.

\section{Experimental Section}

General. All chemicals were purchased from Aldrich and Merck with high-grade quality, and used without any purification. All melting points were obtained by Bamslead Electrothermal 9200 apparatus and are uncorrected. The reactions were monitored by TLC and all yields refer to isolated products. ${ }^{1} \mathrm{H}$ and ${ }^{13} \mathrm{C}$ NMR spectra were recorded in $\mathrm{CDCl}_{3}$ on a Bruker $400 \mathrm{MHz}$ spectrometer. Infrared spectra were recorded on a Bruker FT-IR Equinax-55 spectrophotometer in $\mathrm{KBr}$ with absorption in $\mathrm{Cm}^{-1}$. Elemental analyses were performed using a Carlo Erba EA 1108 instrument. All products were characterized by their spectra and physical data.

General procedure for the synthesis of substituted tetrahydropyridines. To a $25 \mathrm{~mL}$ roundbottom flask were added appropriate benzylamine (1) (1.0 mmol), benzaldehyde (2) $(1.0 \mathrm{mmol})$, two drops acetic acid, acetonitrile $(5.0 \mathrm{~mL})$ and the mixture was stirred vigorously at room temperature. After the benzylamine had been consumed (monitored by TLC), acetonitrile (5.0 $\mathrm{mL})$, dialkyl acetylenedicarboxylate (4) $(1.5 \mathrm{mmol})$ and 2-benzylidenemalononitrile (5) (1.0 mmol) were added. The mixture was then allowed to stir at $50^{\circ} \mathrm{C}$ for $24 \mathrm{~h}$. After completion of the reaction, determined by TLC, the solvent was removed under reduced pressure, and the resulting crude product (72-85\%) was recrystallized from ethanol to give the pure compounds 6a-j.

Dimethyl 1-benzyl-5,5-dicyano-1,4,5,6-tetrahydro-4,6-diphenylpyridine-2,3-dicarboxylate (6a). Mp 146-149 ${ }^{\circ} \mathrm{C}$. IR v/ $\mathrm{cm}^{-1}$ (KBr): 3032, 2952, 2246, 1749, 1703, 1592, 1456, 1434, 1257. ${ }^{1} \mathrm{H}$ NMR (400 MHz, $\left.\mathrm{CDCl}_{3}\right): \delta 3.32\left(3 \mathrm{H}, \mathrm{s}, \mathrm{OCH}_{3}\right.$, Minor isomer), $3.51\left(5 \mathrm{H}, \mathrm{s}, \mathrm{OCH}_{3}\right.$, Major isomer), 3.88 (3H, s, $\mathrm{OCH}_{3}$, Minor isomer), 3.97-4.08 (9H, m, Major/Minor isomers), 4.36-4.54 (6H, m, Major/Minor isomers), 6.98-7.42 (39H, m, ArH, Major/Minor isomers) ppm. ${ }^{13} \mathrm{C}$ NMR $\left(100 \mathrm{MHz}, \mathrm{CDCl}_{3}\right): \delta 29.71,44.31,45.25,47.23,51.59,51.94,53.38,53.41,53.60,54.16$, $59.10,63.29,96.19,112.34,112.61,113.33,113.84,128.23,128.34,128.42,128.50,128.54$, 128.82, 128.95, 129.03, 129.32, 129.55, 130.16, 130.29, 130.63, 131.00, 133.10, 134.19, 135.04, 135.50, 149.84, 149.91, 165.01, 165.19, 165.28, 165.81 ppm. Anal. Calcd for $\mathrm{C}_{30} \mathrm{H}_{25} \mathrm{~N}_{3} \mathrm{O}_{4}$ (491.54): C, 73.30; H, 5.13; N, 8.55. Found: C, 73.18; H, 5.09; N, 8.61.

Dimethyl 1-benzyl-5,5-dicyano-1,4,5,6-tetrahydro-4-(4-methoxyphenyl)-6-phenylpyridine2,3-dicarboxylate (6b). Mp 135-137 ${ }^{\circ} \mathrm{C}$. IR v/ $\mathrm{cm}^{-1}$ (KBr): 3029, 2949, 2245, 1743, 1704, 1593, 
1513, 1457, 1435, 1254. ${ }^{1} \mathrm{H}$ NMR (400 MHz, $\left.\mathrm{CDCl}_{3}\right): \delta 3.45\left(3 \mathrm{H}, \mathrm{s}, \mathrm{OCH}_{3}\right.$, Minor isomer), 3.62 $\left(6 \mathrm{H}, \mathrm{s}, \mathrm{OCH}_{3}\right.$, Major isomer), $3.76\left(3 \mathrm{H}, \mathrm{s}, \mathrm{OCH}_{3}\right.$, Minor isomer $), 3.79\left(6 \mathrm{H}, \mathrm{s}, \mathrm{OCH}_{3}\right.$, Major isomer), $3.97\left(3 \mathrm{H}, \mathrm{s}, \mathrm{OCH}_{3}\right.$, Minor isomer), 4.05-4.16 $(11 \mathrm{H}, \mathrm{m}$, Major/Minor isomers), 4.444.62 (7H, m, Major/Minor isomers), 6.69-7.52 (42H, m, ArH, Major/Minor isomers) ppm. ${ }^{13} \mathrm{C}$ NMR (100 MHz, $\left.\mathrm{CDCl}_{3}\right): \delta 44.56,44.71,46.62,51.69,51.97,53.30,53.35,53.59,54.15$, 55.16 , 55.22, 58.94, 63.14, 96.56, 112.45, 112.77, 113.40, 113.60, 113.83, 126.97, 127.38, $127.96,128.02,128.23,128.35,128.43,128.49,128.82,128.86,128.90,129.28,129.57,130.02$, $130.11,130.40,130.54,130.60,131.06,133.15,134.22,149.55,149.72,159.41,159.96,165.05$, 165.24, 165.32, 165.89 ppm. Anal. Calcd for $\mathrm{C}_{31} \mathrm{H}_{27} \mathrm{~N}_{3} \mathrm{O}_{5}$ (521.56): C, 71.39; H, 5.22; N, 8.06. Found: C, 71.53; H, 5.26; N, 8.04.

Dimethyl 1-benzyl-5,5-dicyano-1,4,5,6-tetrahydro-4-(4-nitrophenyl)-6-phenylpyridine-2,3dicarboxylate (6c). Mp 139-142 ${ }^{\circ} \mathrm{C}$. IR v/cm ${ }^{1}$ (KBr): 3033, 2951, 2248, 1743, 1702, 1593, 1524, 1456, 1435, 1262. ${ }^{1} \mathrm{H}$ NMR (400 MHz, $\left.\mathrm{CDCl}_{3}\right): \delta 3.46\left(3 \mathrm{H}, \mathrm{s}, \mathrm{OCH}_{3}, \mathrm{Minor}\right.$ isomer), 3.58 $\left(10 \mathrm{H}, \mathrm{s}, \mathrm{OCH}_{3}\right.$, Major isomer), $4.02\left(3 \mathrm{H}, \mathrm{s}, \mathrm{OCH}_{3}\right.$, Minor isomer), 4.09-4.22 (18H, m, Major/Minor isomers), 4.49-4.71 (9H, m, Major/Minor isomers), 7.12-8.08 (60H, m, $\mathrm{ArH}$, Major/Minor isomers) ppm. ${ }^{13} \mathrm{C}$ NMR $\left(100 \mathrm{MHz}, \mathrm{CDCl}_{3}\right): \delta 15.29,43.75,44.47,51.83,52.03$, 53.59, 53.76, 53.87, 54.62, 59.54, 65.87, 95.08, 112.22, 112.59, 123.18, 123.73, 128.57, 128.80, $128.95,129.08,129.21,129.25,129.35,129.47,129.55,130.05,130.24,130.43,130.61,130.92$, 132.82, 142.64, 148.22, 150.41, 164.72, 164.77 ppm. Anal. Calcd for $\mathrm{C}_{30} \mathrm{H}_{24} \mathrm{~N}_{4} \mathrm{O}_{6}$ (536.53): C, 67.16; H, 4.51; N, 10.44. Found: C, 67.20; H, 4.52; N, 10.37.

Dimethyl 1-benzyl-5,5-dicyano-1,4,5,6-tetrahydro-6-(4-methoxyphenyl)-4-phenylpyridine2,3-dicarboxylate (6d). Mp 140-142 ${ }^{\circ} \mathrm{C}$. IR v/cm- ${ }^{1}$ (KBr): 3031, 2952, 2242, 1742, 1699, 1591, $1514,1454,1435,1255 .{ }^{1} \mathrm{H}$ NMR $\left(400 \mathrm{MHz}, \mathrm{CDCl}_{3}\right): \delta 3.40\left(3 \mathrm{H}, \mathrm{s}, \mathrm{OCH}_{3}\right.$, Minor isomer), 3.60 $\left(5.4 \mathrm{H}, \mathrm{s}, \mathrm{OCH}_{3}\right.$, Major isomer), $3.82\left(3 \mathrm{H}, \mathrm{s}, \mathrm{OCH}_{3}\right.$, Minor isomer), $3.88\left(5.4 \mathrm{H}, \mathrm{s}, \mathrm{OCH}_{3}\right.$, Major isomer), $3.95\left(3 \mathrm{H}, \mathrm{s}, \mathrm{OCH}_{3}\right.$, Minor isomer), 4.02-4.18 (11H, m, Major/Minor isomers), 4.444.59 (6H, m, Major/Minor isomers), 6.84-7.38 (39H, m, ArH, Major/Minor isomers) ppm. ${ }^{13} \mathrm{C}$ NMR (100 MHz, $\left.\mathrm{CDCl}_{3}\right): \delta$ 44.53, 45.26, 47.57, 51.53, 51.92, 53.29, 53.33, 53.58, 53.90, $55.29,55.37,55.41,58.60,63.38,95.96,112.41,112.75,113.21,113.50,113.82,113.85,114.25$, $114.63,122.18,122.56,128.16,128.28,128.32,128.37,128.53,128.71,128.79,128.91,129.09$, $129.53,129.98,130.38,130.67,133.26,134.48,135.41,135.63,149.99,150.03,160.86,161.19$, 165.03, 165.22, 165.34, 165.82 ppm. Anal. Calcd for $\mathrm{C}_{31} \mathrm{H}_{27} \mathrm{~N}_{3} \mathrm{O}_{5}(521.56)$ : C, 71.39; H, 5.22; N, 8.06. Found: C, 71.79; H, 5.27; N, 8.03.

Dimethyl 1-(4-methoxybenzyl)-4-(2-chlorophenyl)-5,5-dicyano-1,4,5,6-tetrahydro-6-phenylpyridine-2,3-dicarboxylate (6e). Mp 125-128 ${ }^{\circ} \mathrm{C}$. IR v/cm- ${ }^{1}$ (KBr): 3029, 2955, 2240, 1743 , 1701, 1593, 1511, 1451, 1436, 1258. ${ }^{1} \mathrm{H}$ NMR (400 MHz, $\left.\mathrm{CDCl}_{3}\right): \delta 3.37\left(3 \mathrm{H}, \mathrm{s}, \mathrm{OCH}_{3}\right.$, Minor isomer), $3.61\left(5.1 \mathrm{H}, \mathrm{s}, \mathrm{OCH}_{3}\right.$, Major isomer), $3.84\left(3 \mathrm{H}, \mathrm{s}, \mathrm{OCH}_{3}, \mathrm{Minor}\right.$ isomer $), 3.89(5.1 \mathrm{H}, \mathrm{s}$, $\mathrm{OCH}_{3}$, Major isomer), $3.94\left(3 \mathrm{H}, \mathrm{s}, \mathrm{OCH}_{3}\right.$, Minor isomer), 4.09-4.20 (10H, m, Major/Minor isomers), 4.45-4.63 (6H, m, Major/Minor isomers), 6.83-7.44 (35H, m, ArH, Major/Minor isomers) ppm. ${ }^{13} \mathrm{C} \mathrm{NMR}\left(100 \mathrm{MHz}, \mathrm{CDCl}_{3}\right): \delta 40.81,42.97,43.04,51.53,51.59,52.03,53.34$, 53.64, 54.01, 55.38, 55.42, 58.64, 60.21, 63.74, 96.16, 98.63, 102.73, 112.14, 112.39, 113.05, 
$113.18,114.31,114.53,114.67,122.04,122.22,126.74,126.82,127.94,128.30,128.43,128.81$, $128.87,129.02$, 129.60, 129.66, 129.87, 129.97, 130.07, 130.15, 130.78, 133.15, 133.63, 134.21, 134.37, 135.02, 135.46, 150.02, 150.22, 161.03, 161.24, 164.85, 165.11, 165.18, 165.39 ppm. Anal. Calcd for $\mathrm{C}_{31} \mathrm{H}_{26} \mathrm{ClN}_{3} \mathrm{O}_{5}$ (556.01): C, 66.97; H, 4.71; N, 7.56. Found: C, 67.09; H, 4.72; N, 7.53 .

Dimethyl 1-benzyl-4-(4-chlorophenyl)-5,5-dicyano-1,4,5,6-tetrahydro-6-phenylpyridine-2,3-

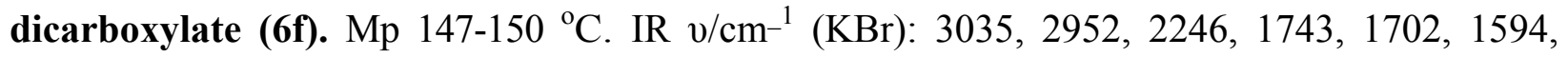
1492, 1457, 1435, 1266. ${ }^{1} \mathrm{H}$ NMR (400 MHz, $\left.\mathrm{CDCl}_{3}\right): \delta 3.46\left(3 \mathrm{H}, \mathrm{s}, \mathrm{OCH}_{3}\right.$, Minor isomer), 3.61 $\left(5.7 \mathrm{H}, \mathrm{s}, \mathrm{OCH}_{3}\right.$, Major isomer), $3.98\left(3 \mathrm{H}, \mathrm{s}, \mathrm{OCH}_{3}\right.$, Minor isomer), 4.06-4.19 (10.6H, m, Major/Minor isomers), 4.45-4.65 (6.7H, m, Major/Minor isomers), 7.07-7.53 (40.6H, m, ArH, Major/Minor isomers) ppm. ${ }^{13} \mathrm{C} \mathrm{NMR}\left(100 \mathrm{MHz}, \mathrm{CDCl}_{3}\right): \delta 29.70,44.15,44.60,46.44,51.71$, 51.98, 53.44, 53.50, 53.65, 54.36, 59.15, 62.85, 95.94, 112.30, 112.49, 113.06, 113.74, 128.39, $128.48,128.60,128.69,128.80,128.86,128.92$, 129.12, 129.28, 129.39, 129.50, 129.71, 129.75 , $129.82,130.06,130.23,130.62,130.74,130.84,133.02,133.60,133.98,134.10,134.28,135.12$, 150.02, 150.10, 164.85, 165.00, 165.05, 165.57 ppm. Anal. Calcd for $\mathrm{C}_{30} \mathrm{H}_{24} \mathrm{ClN}_{3} \mathrm{O}_{4}$ (525.98): C, 68.50; H, 4.60; N, 7.99. Found: C, 68.27; H, 4.53; N, 7.98.

Dimethyl 1-(4-chlorobenzyl)-5,5-dicyano-1,4,5,6-tetrahydro-4,6-diphenylpyridine-2,3dicarboxylate (6g). Mp 130-132 ${ }^{\circ} \mathrm{C}$. IR v/cm- ${ }^{1}$ (KBr): 3030, 2951, 2238, 1737, 1710, 1687 , $1588,1511,1435,1352,1225 .{ }^{1} \mathrm{H}$ NMR $\left(400 \mathrm{MHz}, \mathrm{CDCl}_{3}\right): \delta 3.41\left(3 \mathrm{H}, \mathrm{s}, \mathrm{OCH}_{3}, \mathrm{Minor}\right.$ isomer), $3.57\left(4.8 \mathrm{H}, \mathrm{s}, \mathrm{OCH}_{3}\right.$, Major isomer), $3.92\left(3 \mathrm{H}, \mathrm{s}, \mathrm{OCH}_{3}\right.$, Minor isomer), 4.02-4.15 (10.2H, m, Major/Minor isomers), 4.39-4.57 (5H, m, Major/Minor isomers), 7.05-7.52 (36.4H, $\mathrm{m}$, ArH, Major/Minor isomers) ppm. ${ }^{13} \mathrm{C} \mathrm{NMR}\left(100 \mathrm{MHz}, \mathrm{CDCl}_{3}\right): \delta 29.17,29.37,29.52$, 29.70, 31.93, 47.82, 51.92, 52.11, 52.46, 53.34, 55.41, 58.99, 96.25, 109.65, 126.77, 126.99 , $127.50,127.84,127.97,128.07,128.15,128.27,128.38,128.44,128.51,128.62,128.75,128.84$, 128.90, 129.01, 129.17, 129.67, 133.79, 138.53, 139.10, 152.77, 164.15, 164.21, 164.26, 167.94 ppm. Anal. Calcd for $\mathrm{C}_{30} \mathrm{H}_{24} \mathrm{ClN}_{3} \mathrm{O}_{4}$ (525.98): C, 68.50; H, 4.60; N, 7.99. Found: C, 68.61; H, $4.62 ; \mathrm{N}, 8.04$.

Dimethyl 1-(4-chlorobenzyl)-6-(2-chlorophenyl)-5,5-dicyano-1,4,5,6-tetrahydro-4-(4-nitrophenyl)pyridine-2,3-dicarboxylate (6h). Mp 139-142 ${ }^{\circ} \mathrm{C} . \mathrm{IR} \mathrm{v} / \mathrm{cm}^{-1}(\mathrm{KBr}): 3035,2956,2247$, 1740, 1703, 1593, 1517, 1451, 1434, 1252. ${ }^{1} \mathrm{H}$ NMR (400 MHz, $\left.\mathrm{CDCl}_{3}\right): \delta 3.39\left(3 \mathrm{H}, \mathrm{s}, \mathrm{OCH}_{3}\right.$, Minor isomer), $3.51\left(7.5 \mathrm{H}, \mathrm{s}, \mathrm{OCH}_{3}\right.$, Major isomer), $4.00\left(3 \mathrm{H}, \mathrm{s}, \mathrm{OCH}_{3}, \mathrm{Minor}\right.$ isomer), 4.114.24 (14H, m, Major/Minor isomers), 4.43-4.68 (7.5H, m, Major/Minor isomers), 7.10-8.09 $\left(42 \mathrm{H}, \mathrm{m}, \mathrm{ArH}\right.$, Major/Minor isomers) ppm. ${ }^{13} \mathrm{C} \mathrm{NMR}\left(100 \mathrm{MHz}, \mathrm{CDCl}_{3}\right): \delta 44.12,44.37$, 51.38, 51.97, 52.47, 53.06, 53.49, 54.18, 55.14, 55.46, 58.54, 63.06, 96.76, 112.29, 112.87 , $113.01,113.05,113.44,126.99,127.48,127.98,128.02,128.24,128.30,128.39,128.74,128.82$, $128.96,129.26,129.42,129.79,130.03,130.12$, 130.58, 131.67, 132.10, 133.78, 134.29, 147.58, 148.72, 159.48, 159.64, 163.13, 163.24, 164.31, 164.80 ppm. Anal. Calcd for $\mathrm{C}_{30} \mathrm{H}_{22} \mathrm{Cl}_{2} \mathrm{~N}_{4} \mathrm{O}_{6}$ (605.42): C, 59.52; H, 3.66; N, 9.25. Found: C, 59.73; H, 3.69; N, 9.21.

Diethyl 1-benzyl-6-(2-chlorophenyl)-5,5-dicyano-1,4,5,6-tetrahydro-4-phenylpyridine-2,3-

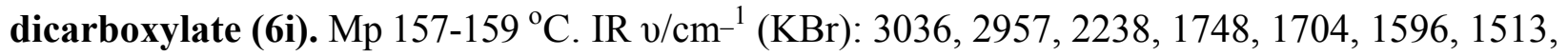


1459, 1431, 1242. ${ }^{1} \mathrm{H}$ NMR (400 MHz, $\left.\mathrm{CDCl}_{3}\right): \delta$ 1.31-1.37 (6H, m, 2CH3, Minor isomer), 1.46$1.50\left(13.2 \mathrm{H}, \mathrm{m}, 2 \mathrm{CH}_{3}\right.$, Major isomer), 4.01-4.13 (8H, m, Major/Minor isomers), 4.21-4.30 (4.8H, m, Major/Minor isomers), 4.36-4.41 (4H, m, 2OCH 2 , Minor isomer), 4.47-4.51 (8.8H, m, $2 \mathrm{OCH}_{2}$, Major isomer), 7.11-7.56 (44.8H, m, ArH, Major/Minor isomers) ppm. ${ }^{13} \mathrm{C}$ NMR (100 $\left.\mathrm{MHz}, \mathrm{CDCl}_{3}\right): \delta 43.19,45.71,48.62,51.72,51.98,53.14,53.76,54.12,54.90,55.12,55.54$, $56.51,58.20,59.24,62.73,63.62,94.41,112.18,112.35,113.31,113.52,113.72,113.95,114.51$, $114.80,122.10,123.51,128.14,128.38,128.42,128.57,128.60,128.74,128.89,128.96,129.17$, $129.43,129.78,130.38,130.87,132.26,134.72$, 135.06, 135.82, 148.31, 153.41, 160.74, 162.52, 165.81, 165.94, 166.12, 166.87 ppm. Anal. Calcd for $\mathrm{C}_{32} \mathrm{H}_{28} \mathrm{ClN}_{3} \mathrm{O}_{4}$ (554.04): C, 69.37; H, 5.09; N, 7.58. Found: C, 69.40; H, 5.07; N, 7.55.

Diethyl 1-(4-chlorobenzyl)-4-(2-chlorophenyl)-5,5-dicyano-1,4,5,6-tetrahydro-6-phenylpyridine-2,3-dicarboxylate (6j). $\mathrm{Mp} 144-147{ }^{\circ} \mathrm{C}$. IR v/cm ${ }^{-1}$ (KBr): 3034, 2957, 2238, 1745 , 1700, 1591, 1513, 1452, 1435, 1250. ${ }^{1} \mathrm{H}$ NMR (400 MHz, $\left.\mathrm{CDCl}_{3}\right): \delta 1.30-1.39\left(6 \mathrm{H}, \mathrm{m}, 2 \mathrm{CH}_{3}\right.$, Minor isomer), 1.44-1.51 (14.4H, m, 2 $\mathrm{CH}_{3}$, Major isomer), 4.02-4.15 (8.6H, m, Major/Minor isomers), 4.22-4.31 (5H, m, Major/Minor isomers), 4.37-4.43 (4H, m, 2OCH 2 , Minor isomer), 4.46-4.53 (9.6H, m, 20CH 2 , Major isomer), 7.04-7.83 (44.2H, m, ArH, Major/Minor isomers) ppm. ${ }^{13} \mathrm{C}$ NMR $\left(100 \mathrm{MHz}, \mathrm{CDCl}_{3}\right): \delta 42.29,43.92,49.66,50.42,51.63,52.14,53.03,54.89$, 55.37, 55.83, 56.24, 56.72, 58.94, 59.20, 61.78, 63.22, 96.72, 112.64, 112.81, 113.17, 113.54, $113.71,115.51,117.52,122.18,123.49,128.11,128.34,128.51,128.65,128.70,128.84,128.88$, $129.13,129.54,129.98,130.14,130.77,133.29,134.12,135.63,136.52,149.74,152.73,161.34$, 161.51, 164.74, 165.33, 165.54, 166.12 ppm. Anal. Calcd for $\mathrm{C}_{32} \mathrm{H}_{27} \mathrm{Cl}_{2} \mathrm{~N}_{3} \mathrm{O}_{4}$ (588.48): C, 65.31; H, 4.62; N, 7.14. Found: C, 65.46; H, 4.66; N, 7.12.

\section{Acknowledgements}

We are thankful to the Office of Graduate Studies of the Vali-e-Asr University of Rafsanjan for partial support of this work.

\section{References}

1. Aridoss, G.; Amirthaganesan, S.; Jeong, Y. T. Bioorg. Med. Chem. Lett. 2010, (20), 2242. http://dx.doi.org/10.1016/j.bmcl.2010.02.015

2. Malviya, M.; Sunil K. Y. C.; Mythri, R. B.; Venkateshappa, C.; Subhash, M. N.; Rangappa, K. S. Bioorg. Med. Chem. Lett. 2009, (17), 5526.

http://dx.doi.org/10.1016/j.bmc.2009.06.032

3. Ishida, J.; Hattori, K.; Yamamoto, H.; Iwashita, A.; Mihara, K.; Matsuoka, N. Bioorg. Med. Chem. Lett. 2005, (15), 4221.

http://dx.doi.org/10.1016/j.bmcl.2005.06.094 
4. Suleman, N. K.; Flores, J.; Tanko, J. M.; Isin, E. M.; Castagnoli, N., Jr. Bioorg. Med. Chem. 2008, (16), 8557.

5. Wimalasena, D. S.; Perera, R. P.; Heyen, B. J.; Balasooriya, I. S.; Wimalasena, K. J. Med. Chem. 2008, (51), 760.

http://dx.doi.org/10.1021/jm070875p

6. Dunbar, P. G.; Durant, G. J.; Rho, T.; Ojo, B.; Huzl, J. J.; Smith, D. A. J. Med. Chem. 1994, (37), 2774.

http://dx.doi.org/10.1021/jm00043a016

7. Chao, Y. X.; He, B. P.; Tay, S. S. W. J. Neuroimmunol. 2009, (216), 39.

http://dx.doi.org/10.1016/j.jneuroim.2009.09.003

8. Roberts, E.; Sanc, J. P.; Sweeney, J. B.; Workman, J. A. Org. Lett. 2003, (5), 4775. Rodinovskaya, L. A.; Shestopalov, A. M.; Chunikihn, K. S. Tetrahedron 2002, (58), 4273.

9. Beeler, A. B.; Gadepalli, R. S. V. S.; Steyn, S.; Castagnoli, N., J.; Rimoldi, J. M. Bioorg. Med. Chem. 2003, (11), 5229.

10. Deskus, J. A.; Epperson, J. R.; Charles, P. S.; Joseph, A. C.; Dextraze, P.; Qian-Cutrone, J.; Gao, Q.; Ma, B.; Beno, B. R.; Mattson, G. K.; Molski, T. F.; Krause, R. G.; Taber, M. T.; Lodge, N. J.; Mattson, R. J. Bioorg. Med. Chem. Lett. 2007, (17), 3099.

http://dx.doi.org/10.1016/j.bmcl.2007.03.040

11. Seyfried, C. A.; Greiner, H. E.; Haase, A. F. Eur. J. Pharmacol. 1989, (160), 31. http://dx.doi.org/10.1016/0014-2999(89)90651-1

12. Jaen, J. C.; Wise, L. D.; Heffner, T. G.; Pugsley, T. A.; Meltzer, L. T. J. Med. Chem. 1988, (31), 1621.

http://dx.doi.org/10.1021/jm00403a022

13. Jaen, J. C.; Caprathe, B. W.; Wise, L. D.; Meltzer, L. T.; Pugsley, T. A.; Heffner, T. G. Bioorg. Med. Chem. Lett. 1993, (3), 639.

http://dx.doi.org/10.1016/S0960-894X(01)81245-2

14. Gwaltney, S. L., II; O’Connor, S. J.; Nelson, L. T. J.; Sullivan, G. M.; Imade, H.; Wang, W.; Hasvold, L.; Li, Q.; Cohen, J.; Gu, W.-Z.; Tahir, S. K.; Bauch, J.; Marsh, K.; Ng, S.C.; Frost, D. J.; Zhang, H.; Muchmore, S.; Jakob, C. G.; Stoll, V.; Hutchins, C. Bioorg. Med. Chem. Lett. 2003, (13), 1359.

http://dx.doi.org/10.1016/S0960-894X(03)00095-7

15. Kamei, K.; Maeda, N.; Katsuragi-Ogino, R.; Koyama, M.; Nakajima, M.; Tatsuoka, T.; Ohno, T.; Inoue, T. Bioorg. Med. Chem. Lett. 2005, (15), 2990.

http://dx.doi.org/10.1016/j.bmcl.2005.04.059

16. Van Voorhis, W. C.; Rivas, K. L.; Bendale, P.; Nallan, L.; Hornéy, C.; Barrett, L. K.; Bauer, K. D.; Smart, B. P.; Ankala, S.; Hucke, O.; Verlinde, C. L. M. J.; Chakrabarti, D.; Strickland, C.; Yokoyama, K.; Buckner, F. S.; Hamilton, A. D.; Williams, D. K.; Lombardo, L. J.; Floyd, D.; Gelb, M. H. J. Med. Chem. 2005, (48), 3704.

17. Hantzsch, A. Chem. Ber. 1881, (14), 1637.

http://dx.doi.org/10.1002/cber.18810140214 
18. Domling, A.; Ugi, I. Angew. Chem., Int. Ed. 2000, (39) 3168.

http://dx.doi.org/10.1002/1521-3773(20000915)39:18<3168::AID-ANIE3168>3.0.CO;2-U

19. Misra, M.; Pandey, S. K.; Pandey, V. P.; Pandey, J.; Tripathi, R.; Tripathi, R. P. Bioorg. Med. Chem. 2009, (17), 625.

http://dx.doi.org/10.1016/j.bmc.2008.11.062

20. Khan, A. T.; Parvin, T.; Choudhary, L. H. J. Org. Chem. 2008, (73), 8398. http://dx.doi.org/10.1021/jo8014962

21. Khan, A. T.; Lal, M.; Khan, M. M. Tetrahedron Lett. 2010, (51), 4419. http://dx.doi.org/10.1016/j.tetlet.2010.06.069

22. Khan, A. T.; Khan, M. M.; Karthi, K. R. B. Tetrahedron 2010, (60), 7762. http://dx.doi.org/10.1016/j.tet.2010.07.075

23. Clarke, P. A.; Zaytzev, P. A.; Whitwood, A. C. Tetrahedron Lett. 2007, (48), 5209. http://dx.doi.org/10.1016/i.tetlet.2007.05.141

24. Mishra, S.; Ghosh, R. Tetrahedron Lett. 2011, (52), 2857. http://dx.doi.org/10.1016/j.tetlet.2011.03.116

25. Hong-Juan, W.; Li-Ping, M.; Zhan-Hui, Z. ACS Comb. Sci. 2011, (13), 181.

26. Ramachandran, R.; Jayanth, S.; Jeong, Y. T. Tetrahedron 2012, (68), 363. http://dx.doi.org/10.1016/j.tetlet.2011.03.116

27. Pal, S.; Singh, V.; Das, P.; Choudhury, L. H. Bioorg. Chem. 2013, (48), 8. http://dx.doi.org/10.1016/j.bioorg.2013.03.003

28. Jiang, H. F.; Li, J. H.; Chen, Z. W. Tetrahedron 2010, (66), 9721. http://dx.doi.org/10.1016/j.tet.2010.10.041

29. Liu, W. B.; Jiang, H. F.; Zhu, S. F.; Wang, W. Tetrahedron 2009, (65), 7985. http://dx.doi.org/10.1016/j.tet.2009.07.046

30. Rodriguez H.; Coro J.; Lam A.; Salfran E.; Rodriguez-Salarichs J.; Suarez M.; Albericio F.; Martin N. Arkivoc 2011, (ix) 125. http://dx.doi.org/10.3998/ark.5550190.0012.909 